\title{
Die Corona-Pandemie in Lateinamerika - ein Überblick aus der Perspektive sozialer Ungleichheiten
}

\author{
Christiane Schulte
}

Die Corona-Pandemie hat Lateinamerika hart getroffen. Die Zahl der Infizierten und der durch Covid-19 verursachten Todesfälle liegt wesentlich höher als in anderen Kontinenten. Die Ursachen dieser besonderen Betroffenheit sind die große soziale Ungleichheit, die in Lateinamerika weltweit am höchsten ist, ein hoher Urbanisierungsgrad, eine schlechte Gesundheitsversorgung und die weit verbreitete Armut. Die Pandemie offenbart bestehende Ungleichheiten und vertieft sie dramatisch. Indigene, Kinder und Jugendliche sowie Frauen sind besonders von der Pandemie und ihren Auswirkungen betroffen.

Soziale Ungleichheiten sind die Ursache von Armut und Hunger, verletzen die Menschenwürde und Menschenrechte, befördern soziale Konflikte und Gewalt in der Gesellschaft und erhöhen die Vulnerabilität gegenüber Pandemien wie Corona (Brot für die Welt 2019). Im Folgenden gebe ich einen Überblick über das Pandemiegeschehen in Lateinamerika unter besonderer Berücksichtigung von sozialen Ungleichheiten. Der Beitrag ist folgendermaßen aufgebaut: Im ersten Abschnitt stelle ich aktuelle Zahlen zu Infektionsraten, Todeszahlen und Letalität vor. Ich beziehe mich dabei vor allem auf Daten der WHO. Nachfolgend diskutiere ich, wie sich die besondere Verwundbarkeit der Region gegenüber der Pandemie erklären lässt und gehe anschließend auf unterschiedliche Dimensionen sozialer Ungleichheit ein, die als Risikofaktoren verstanden werden können: Einkommensungleichheit, Ethnizität, Alter und Geschlecht.

\section{Die Corona-Gesundheitskrise in Lateinamerika}

Ende Oktober 2021 beträgt die Anzahl der Corona-Infizierten in Lateinamerika mehr als 45,5 Millionen Menschen. Seit dem Ausbruch der Pandemie auf dem Kontinent im Februar 2020 sind insgesamt 1,5 Millionen Menschen an Corona gestorben. Lateinamerika hat einen Anteil von 19 Prozent an den weltweiten Corona-Fällen und von knapp 31 Prozent an den weltweit durch Corona verstorbenen Menschen. Vor dem Hinter- 
grund, dass die Bevölkerung in Lateinamerika und der Karibik nur 8,4 Prozent der Weltbevölkerung ausmacht, wird das Ausmaß der Gesundheitskrise deutlich. Besonders gravierend ist die hohe Letalität, die Zahl der Verstorbenen verglichen mit den Covid-19-Erkrankten, die im Juni 2021 in Lateinamerika mit 3,44 so hoch war wie in keiner anderen Region. Der weltweite Durchschnitt betrug 2,17.

Die Pandemie hat sich in den einzelnen Ländern der Region jeweils unterschiedlich entwickelt. Ende Oktober 2021 sind laut WHO in absoluten Zahlen die meisten Corona-Erkrankten in Brasilien, Argentinien, Kolumbien und Mexiko zu verzeichnen, die meisten mit Corona im $\mathrm{Zu}$ sammenhang stehenden Todesfälle gibt es in Brasilien, Mexiko, Peru und Kolumbien.

Umgerechnet auf 100.000 Einwohner*innen ergibt sich folgendes Bild: Die meisten Menschen haben sich in Uruguay, Argentinien, Brasilien und Chile mit Corona angesteckt, die meisten Todesfälle wurden in Peru und Brasilien registriert. Die Letalität ist in Peru und Mexiko mit Abstand am höchsten, diese Länder liegen mit 9,1 Prozent und 7,6 Prozent an der Spitze, gefolgt von Ecuador mit 6,4 und Bolivien mit 3,7 Prozent. Die Zahlen vermitteln eine Idee davon, wie in dem jeweiligen Land mit der Corona-Krise umgegangen werden konnte.

Im September 2021 sind vier verschiedene Mutationen weltweit im Umlauf (Alfa, Beta, Gamma und Delta). Eine von ihnen, Gamma, wurde in Brasilien zuerst entdeckt. In den meisten Ländern der Region sind bis September 2021 Alfa, Beta und Delta im Umlauf. Weitere Mutationen, die derzeit noch als nicht so gefährlich eingestuft werden, wurden in Brasilien (Zeta) und Peru (Lambda) entdeckt (OPS 2021: 10f.).

Die WHO berichtet zudem von einer Lungenkrankheit, die bei Kindern und Jugendlichen auftritt, dem sogenannten Sindrome Inflamatorio multisistemico, SIM-P. Insgesamt sind bis September 2021 in der Region 7547 Fälle aufgetreten, allein in Brasilien 1269, von denen 153 gestorben sind (OPS 2021: 20).

\section{Risikofaktoren in der Corona-Pandemie}

Die CEPAL hat als einen Risikofaktor für die Ansteckung mit Covid-19 die starke Urbanisierung der lateinamerikanischen Gesellschaften identifiziert (CEPAL 2021: 16 ff.). Das Corona-Virus hat sich weltweit insbesondere in Kontexten hoher Bevölkerungsdichte ausgebreitet. Im Juli 2020 lebten weltweit mehr als 90 Prozent aller Corona-Infizierten in Städten (CEPAL 2021: 16). 
Lateinamerika ist die am stärksten urbanisierte Region der Welt. 81 Prozent der Bevölkerung lebt in Städten und 35 Prozent davon in urbanen Metropolen mit mehr als einer Million Einwohner*innen. Auf dem Kontinent befinden sich zudem fünf so genannte Megastädte mit mehr als zehn Millionen Einwohner*innen. ${ }^{1}$ In Lateinamerika verbindet sich der hohe Urbanisierungsgrad mit einer starken Wohnsegregation. Arme und Reiche leben in getrennten Stadtteilen mit sehr unterschiedlichen Infrastrukturausstattungen. Die städtischen Haushalte von Menschen in Armut und extremer Armut leiden überdurchschnittlich stark unter engen Wohnverhältnissen. 22 Prozent der in Armut lebenden Bevölkerung hat keinen $\mathrm{Zu}$ gang zu fließendem Wasser, 34 Prozent lebt ohne Internetanschluss und 45 Prozent ohne eigenes Bankkonto. Zwei Drittel der Armen leben in Städten (Lustig/Tommasi 2020: 286; CEPAL 2021: 18 f.). Für sie ist unter den gegebenen Wohnverhältnissen die soziale Distanzierung und regelmäBiges Händewaschen nur schwer möglich - ihre Vulnerabilität gegenüber der Pandemie ist daher extrem hoch.

Ein weiterer Risikofaktor in der Corona-Pandemie ist der mangelhafte Zugang zur Gesundheitsversorgung und die Qualität der Gesundheitssysteme selbst. Dies betrifft insbesondere Menschen, die im informellen Sektor tätig sind oder in ländlichen Regionen leben. Vor der Corona-Pandemie waren nur 60,5 Prozent der Bevölkerung Lateinamerikas gesundheitsversichert (CEPAL 2021: 31). Mit 64,8 Prozent sind die städtischen Einwohner*innen häufiger versichert als Menschen im ländlichen Raum (43,1 Prozent).

Die öffentlichen Gesundheitssysteme in Lateinamerika sind chronisch unterfinanziert. Die staatlichen Ausgaben für Gesundheit sind mehrheitlich weit von den von der WHO empfohlenen sechs Prozent des Bruttoinlandsprodukts entfernt (CEPAL 2021: 19). Die Krankenhäuser können in vielen Ländern nur unzureichend auf die steigenden Bedarfe durch die Corona-Pandemie reagieren. Bei der Ausstattung der Krankenhäuser mit Betten und Personal schneiden Guatemala, Haiti, Honduras, Nicaragua und Venezuela sehr schlecht ab, die obere Spitze bildet Kuba, gefolgt von Chile und Brasilien (CEPAL 2021: 20). Viele Menschen sterben, weil sie während der Corona-Pandemie keinen Platz im Krankenhaus bekommen.

Betroffen von den negativen Auswirkungen der schwachen Gesundheitssysteme sind Menschen mit niedrigem Einkommen und informellen Arbeitsverhältnissen sowie Arbeitslose, Migrant*innen, Kinder und Alte, die sich keine private Gesundheitsversorgung leisten können. In der Pan-

1 Mexiko-Stadt, Lima, Buenos Aires, Río de Janeiro und São Paulo. 
demie müssen notwendige Gesundheitsausgaben von den nicht-versicherten Haushalten selbst getragen werden und belasten die ohnehin niedrigen Haushaltseinkommen unverhältnismäßig. Dies führt dazu, dass Erspartes aufgebraucht wird oder Kredite aufgenommen werden müssen.

Die Pandemie hat ebenfalls die unzureichende Altersversorgung in großen Teilen der Bevölkerung deutlich gemacht. Rund ein Viertel der Menschen über 65 Jahre sind nach Zahlen der CEPAL für 2019 ohne Altersversicherung in die Corona-Pandemie geraten, darunter mehr Frauen als Männer. Aufgrund des hohen Risikos für ältere Menschen ist es für sie besonders schwierig ihre Grundbedürfnisse zu befriedigen. Viele nicht-versicherte ältere Menschen leben in Mehrgenerationenhaushalten mit den oben beschriebenen Risiken der engen Wohnverhältnisse und dem daraus folgenden höheren Ansteckungsrisiko. 2019 waren etwa 60 Prozent der Menschen im arbeitsfähigen Alter zwischen 15 und 64 Jahren nicht rentenversichert (CEPAL 2021: 121ff.).

\section{Wirtschafts- und soziale Krise: Zunahme von Armut und extremer Armut}

Menschen, die in Armut und extremer Armut leben, sind stärker von den aufgeführten Risikofaktoren betroffen. Sie leben in engeren Wohnverhältnissen und sind weniger häufig sozialversichert - Armut begünstigt somit eine Ansteckung mit Corona. Von Armut betroffene Menschen haben größere Schwierigkeiten Präventionsmaßnahmen wie Homeoffice, Social Distancing oder andere Hygienemaßnahmen umzusetzen. Gleichzeitig sind sie stärker von Arbeitsplatzverlust und Einkommenseinbußen betroffen. So hat die Corona-Pandemie nicht nur direkte Auswirkungen auf die Gesundheit der Menschen, sondern auch indirekte Auswirkungen auf andere Lebensbereiche und führt zu einer Zunahme von Armut.

Die Corona-Pandemie wird in Lateinamerika von einer Wirtschaftskrise und einer sozialen Krise begleitet. Im ersten Pandemiejahr 2020 ist das Wirtschaftswachstum gemessen am BIP um 7,7 Prozent zurückgegangen. Besonders betroffen sind Venezuela, Peru, Argentinien und Panamá mit einem Rückgang des BIP um 10 bis 30 Prozent (CEPAL 2020: 49). Bereits vor der Pandemie hatte sich das Wirtschaftswachstum in Lateinamerika verlangsamt und zwischen 2014 und 2019 jährlich nur 0,3 Prozent betragen. Gleichzeitig ist die Armut erneut angestiegen. Die CEPAL schätzt einen Anstieg der extremen Armut von 11,3 Prozent in 2019 auf 12,5 Prozent in 2020 und den der Armut von 19,1 auf 21,2 Prozent. So sind im Jahr 2020 weitere 22 Millionen Menschen in Armut geraten und acht Millionen in extreme Armut (CEPAL 2021: 15). 
Damit setzt sich ein Trend fort, der schon vor der Pandemie begonnen hat. Der Anteil der von Armut betroffenen Bevölkerungsgruppen hat sich bereits zwischen 2014 und 2019 von 27,8 auf 30,5 Prozent vergrößert, der der extremen Armut von 7,8 auf 11,3 Prozent (CEPAL 2021). Haushalte von Alleinerziehenden mit Kindern und Haushalte, in denen drei Generationen zusammen leben sind mit 33,4 bzw. 35,1 Prozent stärker von Armut betroffen als Paare ohne Kinder (9,5 Prozent) oder Einpersonenhaushalte (10,4 Prozent) (CEPAL 2021: 61).

\section{Soziale Ungleichheit vor der Corona-Pandemie}

Die Ausmaße von Armut und ihre Konzentration in bestimmten gesellschaftlichen Gruppen verweisen auf die strukturell verankerte wirtschaftliche und soziale Ungleichheit, die sich bereits vor der Corona-Pandemie abzeichnete.

„Soziale Ungleichheit bezeichnet die ungleiche Verteilung von und die ungleichen Zugangschancen zu materiellen und immateriellen Ressourcen und sich daraus ergebende, dauerhaft und unfreiwillig unterschiedlichen Möglichkeiten zur gesellschaftlichen, wirtschaftlichen, kulturellen und politischen Teilhabe und zur Verwirklichung der persönlichen Potenziale und Rechte“ (Brot für die Welt 2019: 4).

Dabei verstärken sich verschiedene Dimensionen der Benachteiligung gegenseitig: die wirtschaftliche Ungleichheit, der ungleiche Zugang zu Dienstleistungen im Bildungs- und Gesundheitsbereich und daraus folgende ungleiche Ernährungs-, Gesundheits- und Bildungsstatus sowie Lebenserwartung und eine ungleiche politische Teilhabe und Macht (Brot für die Welt 2019: 7).

Die Ursachen liegen unter anderem in der ungleichen Verteilung von Einkommen und Vermögen. Nach den Daten des von Thomas Piketty und anderen Wissenschaftler*innen gegründeten World Inequality Lap verfügten 2019 in Lateinamerika 10 Prozent der Bevölkerung über einen Anteil von 55,7 Prozent am Gesamteinkommen und 50 Prozent der Bevölkerung nur über einen Anteil von 9,9 Prozent. Besonders ungleich ist die Einkommensverteilung in Brasilien, Chile, Peru, Mexiko und Costa Rica. Weniger ungleich verteilt sind die Einkommen in Kuba und Ecuador (WID 2019).

Im Bericht zur weltweiten Ungleichheit von 2018 stellen die Wissenschaftler*innen fest, dass die Einkommensungleichheit in den letzten 40 Jahren weltweit zugenommen hat. In Lateinamerika hat das reichste 1 
Prozent der Bevölkerung seinen Anteil am Gesamteinkommen von 23,4 auf 26,7 Prozent erhöhen können (s. Burchardt in diesem Band)

„Hauptursache der ökonomischen Ungleichheit ist die ungleiche Verteilung von Kapital, das sich entweder in privater oder öffentlicher Hand befinden kann. Wir zeigen, dass seit 1980 in fast allen Ländern (...) riesige Mengen an öffentlichem Vermögen in private Hände transferiert wurden." (World Inequality Lab 2018: 10)

Die Umwandlung von öffentlichem in privates Vermögen in den letzten 40 Jahren hat die Vermögensungleichheit zwischen den Menschen erheblich vergrößert. Gleichzeitig hat sich der Spielraum der Regierungen, der Ungleichheit durch sozialpolitische Maßnahmen entgegenzuwirken, verringert (World Inequality Lab 2018: 10ff.).

Brot für die Welt bezeichnet Ungleichheit als „Resultat von Machtverhältnissen, politischen Entscheidungen und daraus resultierenden Institutionen“" (Brot für die Welt 2019: 9). Die Verteilung und Besteuerung von Einkommen und Vermögen, die Entscheidung über Export- und Importpolitik, Löhne und Gehälter, Zugang zu Krediten, aber auch soziale Sicherungssysteme, öffentliche Daseinsfürsorge, Zugangsbedingen zu Bildung und Gesundheit werden in politischen Systemen und Prozessen entschieden. Ungleichheit zwischen gesellschaftlichen Gruppen ist eine Ursache von sozialen Konflikten bzw. verschärft diese (Brot für die Welt 2019: 6).

\section{Soziale Ungleichheit in der Pandemie: Ethniziät, Alter und Geschlecht}

Indigene sowie Kinder, Jugendliche und Frauen sind von Armut und Ernährungsunsicherheit, informellen Arbeitsverhältnissen, schlechten Wohnverhältnissen und mangelnder Gesundheitsversorgung überdurchschnittlich stark betroffen. ${ }^{2}$

\section{Ethnizität}

In Lateinamerika gehören etwa 50 Millionen Menschen einer der ca. 500 verschiedenen ethnischen Gruppen an. Obwohl sie nur 8 Prozent der Gesamtbevölkerung repräsentieren, haben sie einen Anteil von 14 Prozent

2 Ebenfalls betroffen sind Flüchtlinge und Migrant*innen. Auch zu Afrolateinamerikaner*innen fehlen belastbare Zahlen. 
an der in Armut lebenden Bevölkerung und einen Anteil von 17 Prozent an der extremen Armut (Lustig/Tommasi 2020: 288). Von allen indigenen Menschen leben 46,7 Prozent in Armut und 17,3 Prozent in extremer Armut (CEPAL 2021: 61). Bei der afrolateinamerikanischen Bevölkerung liegt der Anteil der in Armut bzw. in extremer Armut lebenden Menschen bei 25,8, bzw. 7,8 Prozent (CEPAL 2021: 62).

Für die indigene Bevölkerung wurden bis Ende September 2021 633.888 Corona-Infizierungen und 15.752 in Zusammenhang mit Covid-19 aufgetretene Todesfälle bei der WHO gemeldet. Besonders auffällig ist die hohe Letalität in den indigenen Gemeinden in Mexiko. 15 Prozent der an Covid-19 erkrankten Indigenen sind im Zusammenhang mit der Krankheit gestorben. Nicht für alle Länder liegen jedoch Informationen vollständig vor.

Indigene Gruppen in Lateinamerika sind in der Pandemie durch prekäre Wohnverhältnisse einem besonders hohen Risiko ausgesetzt. In den fünf Ländern, in denen 80 Prozent der indigenen Bevölkerung lebt (Chile, Kolumbien, Guatemala, Mexiko und Peru), hat ein großer Teil keinen Zugang zu fließendem Wasser für das regelmäßige Händewaschen oder keinen Zugang zu Wasser- und Abwasserversorgung. Das gilt für sieben von zehn indigenen Personen in Guatemala, für sechs von zehn indigenen Personen in Peru, für 50 Prozent der Indigenen in Kolumbien und für 20 Prozent der Indigenen in Mexiko (CEPAL 2021: 18). Besonders kritisch ist die Situation für Indigene in Guatemala und Kolumbien.

\section{Kinder und Jugendliche}

Kinder und Jugendliche sind laut CEPAL einem durch die Pandemie ausgelösten Teufelskreis aus Armut und schlechtem Gesundheits- und Bildungszustand ausgesetzt. Schon vor der Pandemie sind Kinder und Jugendliche mehr als andere Altersgruppen von Armut betroffen. Je jünger die Bevölkerung ist, desto größer ist ihr Armutsrisiko. 47 Prozent der Kinder bis 14 Jahren und 33,5 Prozent der Jugendlichen zwischen 15 und 24 Jahren sind von Armut betroffen (CEPAL 2021: 62).

Obwohl Kinder und Jugendliche von Covid-19 und schweren Krankheitsverläufen erheblich weniger stark als andere Altersgruppen betroffen sind, sind sie aufgrund der wirtschaftlichen und sozialen Auswirkungen auf arme Haushalte, in denen sie mehrheitlich leben, ganz wesentlich Opfer der Krise (CEPAL 2021: 14). Die Pandemie hat auch in Lateinamerika zu einer massiven Schließung der Schulen und Universitäten geführt. Davon waren bis Ende 2020 bereits 165 Millionen Schüler*innen und Stu- 
dierende betroffen (CEPAL 2021: 22). Kinder und Jugendliche aus Haushalten mit niedrigerem Einkommen haben einen schlechteren Zugang zu digitalen Technologien und damit weniger Möglichkeiten, am digitalen Unterricht teilzunehmen. Sie sind in der Folge stärker von Bildungslücken betroffen. Eine Umfrage in Argentinien hat ergeben, dass Schüler*innen in Haushalten von Akademiker*innen zu 73,6 Prozent ihre Hausaufgaben täglich erledigten, während dies in Haushalten der Arbeiterklasse nur zu 51 Prozent geschieht (CEPAL 2021:28; Fußnote 14). Kinder aus ärmeren Haushalten sind auch stärker von dem Risiko eines Schulabbruchs betroffen. Nach Schätzungen der CEPAL gilt das aktuell für 3 Millionen Kinder und Jugendliche in der Region (CEPAL 2021: 24).

Die Schulschließungen haben zudem Auswirkungen auf die Ernährung und Gesundheit der Kinder und Jugendlichen. Die Schulspeisungsprogramme, für viele Kinder die wichtigste Mahlzeit am Tag, sind stark eingeschränkt worden. Auch Programme der Sexualerziehung und reproduktiven Rechte, einschließlich der Verteilung von Verhütungsmitteln, fallen aus. Das wirkt sich besonders nachteilig auf die Mädchen aus, die zuhause durch die Pandemie außerdem stärker sexuellen Übergriffen und intrafamiliärer Gewalt ausgesetzt sind und zusätzliche Haus- und Pflegearbeiten übernehmen müssen. Die Pandemie vergrößert so die soziale Ungleichheit zwischen Kindern und Jugendlichen aus unterschiedlichen gesellschaftlichen Gruppen sowie zwischen Jungen und Mädchen. Es wird auf die langfristigen Folgen verwiesen, die eine Krise wie die Corona-Pandemie für das weitere Leben der Kinder und Jugendlichen haben können. Unterernährung, Schulabbruch und traumatische Erfahrungen haben häufig irreversible Effekte (Lustig/Tommasi 2020: 285).

\section{Geschlecht}

Die Corona-Pandemie hat sich auch auf das Leben von Frauen und Männern unterschiedlich ausgewirkt und die Benachteiligung von Frauen gegenüber Männern verstärkt. Auch sind Frauen vergleichsweise schlecht durch die Pandemie gekommen. Die Armutsrate für Frauen im wirtschaftlich aktiven Alter war bereits 2019 um 12,7 Prozent höher als für Männer (CEPAL 2021: 52).

In der Geschlechtergerechtigkeit lag Lateinamerika nach Daten von 2019 mit einem Wert von 25,4 (Social Institutions and Gender Index, SIGI) hinter Europa und Nordamerika, aber vor Afrika und Asien (40,3 und 35,99) (Vgl. OECD 2020). Von den vier im Index integrierten Aspekten schneidet die Region besonders schlecht bei der Diskriminierung in 
der Familie ab: die Verteilung von Arbeiten und Verantwortlichkeiten im Haushalt, intrafamiliäre Diskriminierung und eine hohe Zahl von Kinderehen sind besorgniserregend.

Lateinamerika ist weltweit die einzige Region, in der es seit 1990 keine signifikanten Veränderungen bei den Kinderehen gibt. Eins von sechs Mädchen geht vor dem 18. Lebensjahr eine Ehe oder eheähnliche Beziehung ein (OECD 2020: 21). 39 Prozent dieser Kinderehen finden in den 20 Prozent der ärmsten Haushalte statt. Die Corona-Pandemie hat die Situation von Frauen auf unterschiedlichen Ebenen verschlechtert (OECD 2020: 23ff.):

- Frauen sind im informellen Sektor überrepräsentiert (Dienstleistungsbereich, Verarbeitung) und sind besonders von den Ausgangsbeschränkungen betroffen. 80 Prozent der privaten Haushaltshilfen sind Frauen.

- Frauen stellen mit zwei Dritteln die große Mehrheit der Arbeitnehmer*innen im Gesundheitsbereich. 86 Prozent der Pflegekräfte sind Frauen, die einem großen Infektionsrisiko ausgesetzt sind.

- Die Corona-Pandemie und die verhängten Ausgangsbeschränkungen haben die unbezahlte Haus- und Pflegearbeit massiv erhöht. Schon vor Corona haben Frauen dreimal so viel Zeit dafür verwandt als Männer (s. Kim in diesem Band).

- Expert*innen gehen davon aus, dass analog zu anderen Pandemien auch während Corona die intrafamiliäre Gewalt gegenüber Frauen und Mädchen steigt. Bereits vor der Corona-Pandemie haben 15 Prozent der Frauen über häusliche Gewalt geklagt (Lustig/Tommasi 2020: 288).

- Der Zugang zu reproduktiven Rechten und Dienstleistungen hat sich für Frauen erheblich verschlechtert.

Aus diesen Situationen leitet sich ein höheres Risiko für eine Corona-Infektion, eine Zunahme der unbezahlten Arbeit und der Gewalt gegenüber Frauen ab (s. Kim in diesem Buch).

\section{Fazit}

Die Corona-Pandemie hat wie unter der Lupe die wirtschaftlichen und sozialen Ungleichheiten in Lateinamerika sichtbar gemacht. Armut, mangelhafte Gesundheitsversorgung und defizitäre Wohnverhältnisse sowie geringe Bildungschancen betreffen Frauen, Kinder und Jugendliche sowie indigene Gruppen besonders stark und verletzen ihre Menschenrechte. Sie können sich schlechter gegen die Ansteckung mit Krankheiten wie Corona schützen, sind im Krankheitsfall weniger gerüstet, um der Krankheit 
zu begegnen und selbst ohne Infizierung kommen sie schlechter durch die durch Corona ausgelöste wirtschaftliche und soziale Krise als andere Bevölkerungsgruppen.

In den meisten lateinamerikanischen Ländern haben die Regierungen neben Maßnahmen der sozialen Distanzierung, Ausgangssperren und Quarantäne auch soziale Schutzmaßnahmen und Hilfsprogramme für ärmere und gefährdete Haushalte implementiert. Für das Jahr 2020 hat die CEPAL insgesamt 263 beitragsfreie Hilfsmaßnahmen registriert (CEPAL 2021: 32). Monetäre Transferleistungen sowie Medikamente und Hilfsleistungen erreichten im Durchschnitt 49,4 Prozent der Bevölkerung in der Region. Damit sind in der Krise die öffentlichen Sozialausgaben der Regierungen zwar gestiegen. ${ }^{3}$ Diese Maßnahmen haben nicht verhindern können, dass die Schere zwischen Arm und Reich weiter auseinandergeht. Benedicte Bull und Franciso Robles Rivera (2020) stellen in ihrer Untersuchung fest, dass selbst während der Corona-Pandemie eine Umverteilung zugunsten der reichsten Gesellschaftsschichten stattgefunden hat. Allein der Reichtum der 73 reichsten Lateinamerikaner hat sich im Jahr 2020 um 17 Prozent erhöht (Bull/Robles Rivera 2020: 85; s. Burchardt in diesem Buch).

Die Regierungen der Region sind damit nicht nur in der Pflicht, ihre Bevölkerung in der Corona-Pandemie besser gegen die Krankheit zu schützen, sondern darüber hinaus über strukturelle Reformen Einkommens-, Bildungs- und Gesundheitsgerechtigkeit nachhaltig zu sichern. Die internationale Gemeinschaft ist aufgerufen, weltweit Impfgerechtigkeit herzustellen. Möglich ist das über eine umfassende, temporäre Freigabe der Patente, wie von Südafrika und Indien 2020 bei der WTO beantragt worden. Der TRIPS Waiver umfasst die zeitweise Aussetzung der Rechte des geistigen Eigentums auf Diagnostika, Therapeutika und Impfstoffe gegen Covid-19 und schließt damit auch Schnelltests und Beatmungsgeräte ein (Brot-für-die-Welt.de). Dahinter steckt auch die Ansicht, dass die Pandemie nur weltweit oder gar nicht eingedämmt werden kann, sonst kommt es zu weiteren aggressiven Mutationen, die sich weltweit verbreiten. Die OECD warnt vor Trillionen schweren Schäden, wenn ärmere Länder länger vom Zugang zu Impfstoffen ausgeschlossen werden. Der Zugang zu Impfstoffen für alle Menschen in allen Ländern ist ein moralisches und menschenrechtliches Gebot.

3 Vor der Pandemie betrugen sie im Durchschnitt 11,5 Prozent des Bruttoinlandsprodukts, mit großen Unterschieden zwischen den einzelnen Ländern. 


\section{Literatur}

Acemoglu, Daron; Robinson, James A. (2015): Warum Nationen scheitern. Die Ursprünge von Macht, Wohlstand und Armut, Frankfurt am Main: Fischer.

Bárcena, Alicia; Cimoli, Mario (2020): Asimetrías estructurales y crisis sanitaria: el imperativo de una recuperación transformadora para el desarrollo sostenible en América Latina y el Caribe, in: Revista de la CEPAL, 132, 17-46.

Blofield, Merike; Hoffmann, Bert (2020): Lateinamerikas sozialpolitische Antworten auf die COVID-19-Krise, in: GIGA FOKUS Lateinamerika, 07, [https://www.g iga-hamburg.de/de/publikationen/giga-focus/lateinamerikas-sozialpolitische-ant worten-auf-die-covid-19-krise], letzter Aufruf 30.12.2021.

Brot für die Welt (2019): Policy. Die Kluft zwischen Arm und Reich überwinden. Soziale Ungleichheit als entwicklungspolitische Herausforderung. Berlin.

Brot-für-die-Welt.de (2021): Warum Corona für arme Länder besonders gefährlich ist, in: [Brot-für-dieWelt.de, brot-fuer-die-welt.de/themen/corona/], letzter Aufruf 12.10.2021.

Bull, Benedicte; Robles Rivera, Francisco (2020): El COVID-19, las élites y el futuro de la economía política de la reducción de la desigualdad en América Latina, in: Revista de la CEPAL, 132, 79-94.

CEPAL (2020): Balance Preliminar de las Economías de America Latina y el Caribe, Santiago de Chile: CEPAL

CEPAL (2021): Panorama Social de América Latina, Santiago de Chile: CEPAL

Lustig, Nora; Tommasi, Mariano (2020): El COVID-19 y la protección social de los grupos pobres y vulnerables en América Latina: un marco conceptional, in: Revista de la CEPAL, 132, 283-295.

OECD (2020): SIGI 2020 Regional Report for Latin America and the Caribbean, Social Institutions and Gender Index, Paris: OECD Publishing.

Organización Panamericana de la Salud, OPS (2021): Actualización Epidemiológica Enfermedad por coronavirus (COVID-19) vom 27.09.2021, [https://www.paho.org/ es/documentos/actualizacion-epidemiologica-enfermedad-por-coronavirus-covid -19-27-septiembre-2021], letzter Aufruf 16.10.2021.

UNDP (2020): Human Development Report 2020. The next frontier. Human development and the Antropocene, New York: UNDP

World Health Organization (2021): WHO Coronavirus (COVID-19) Dashboard, laufend aktualisierte Datenbank, [https://covid19.who.int], letzter Aufruf 12.10.2021.

World Inequality Lab (2018): Bericht zur weltweiten Ungleichheit, Kurzfassung. Deutsche Fassung.

World Inequality Database (2021): Top $10 \%$ national income share, auf: wid.word vom 29.05.2021 (laufend aktualisierte Datenbank), https://wid.world/world/\#spt inc_p90p100_z/US;FR;DE;CN;ZA;GB;WO/2019/eu/k/p/yearly/s/false/24.322/80/ curve/false/country], letzter Aufruf 12.10.2021. 
